\title{
The Advancing Understanding of Transportation Options (AUTO) study: design and methods of a multi-center study of decision aid for older drivers
}

Marian E. Betz ${ }^{1,2^{*}}$ (D) Faris Omeragic ${ }^{1}$, Lauren Meador ${ }^{1}$, Carolyn G. DiGuiseppi ${ }^{3}$, Nicole R. Fowler ${ }^{4}$, S. Duke Han ${ }^{5}$, Linda Hill ${ }^{6}$, Rachel L. Johnson ${ }^{7}$, Christopher E. Knoepke ${ }^{8,9}$, Daniel D. Matlock ${ }^{2,8,10}$, Ryan Moran ${ }^{6}$ and on behalf of the AUTO Research Team

\begin{abstract}
Background: Decision-making about when to stop driving for older adults involves assessment of driving risk, availability of support or resources, and strong emotions about loss of independence. Although the risk of being involved in a fatal crash increases with age, driving cessation can negatively impact an older adult's health and well-being. Decision aids can enhance the decision-making process by increasing knowledge of the risks and benefits of driving cessation and improve decision quality. The impact of decision aids regarding driving cessation for older adults is unknown.

Methods: The Advancing Understanding of Transportation Options (AUTO) study is a multi-site, two-armed randomized controlled trial that will test the impact of a decision aid on older adults' decisions about changes in driving behaviors and cessation. AUTO will enroll 300 drivers age $\geq 70$ years with a study partner (identified by each driver); the dyads will be randomized into two groups ( $n=150$ /group). The decision aid group will view the webbased decision aid created by Healthwise at baseline and the control group will review information about driving that does not include evidence-based elements on risks and benefits and values clarification about driving decisions. The AUTO trial will compare the effect of the decision aid, versus control, on a) immediate decision quality (measured by the Decisional Conflict Scale; primary outcome); b) longitudinal psychosocial outcomes at 12 and 24 months (secondary outcomes); and c) longitudinal driving behaviors (including reduction or cessation) at 12 and 24 months (secondary outcomes). Planned stratified analyses will examine the effects in subgroups defined by cognitive function, decisional capacity, and readiness to stop driving.

(Continued on next page)
\end{abstract}

\footnotetext{
* Correspondence: Marian.Betz@cuanschutz.edu

'Department of Emergency Medicine, School of Medicine, University of Colorado Anschutz Medical Campus, Aurora, CO, USA

${ }^{2}$ VA Eastern Colorado Geriatric Research Education and Clinical Center, Aurora, CO, USA

Full list of author information is available at the end of the article
}

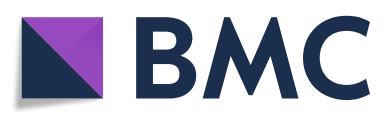

(- The Author(s). 2021 Open Access This article is licensed under a Creative Commons Attribution 4.0 International License, which permits use, sharing, adaptation, distribution and reproduction in any medium or format, as long as you give appropriate credit to the original author(s) and the source, provide a link to the Creative Commons licence, and indicate if changes were made. The images or other third party material in this article are included in the article's Creative Commons licence, unless indicated otherwise in a credit line to the material. If material is not included in the article's Creative Commons licence and your intended use is not permitted by statutory regulation or exceeds the permitted use, you will need to obtain permission directly from the copyright holder. To view a copy of this licence, visit http://creativecommons.org/licenses/by/4.0/. The Creative Commons Public Domain Dedication waiver (http://creativecommons.org/publicdomain/zero/1.0/) applies to the data made available in this article, unless otherwise stated in a credit line to the data. 
(Continued from previous page)

Discussion: The AUTO study is the first large-scale randomized trial of a driving decision aid for older adults. Results from this study will directly inform clinical practice about how best to support older adults in decision-making about driving.

Trial registration: ClinicalTrials.gov: NCT04141891. Registered on October 28, 2019. Located at https://clinicaltrials. gov/ct2/show/NCT04141891

Keywords: Older driver, Driving, Decision-making, Motor vehicle, Randomized trial, Geriatric, Decision aid

\section{Background}

There are 44 million licensed drivers aged $\geq 65$ years in the United States (CDC 2020) and driving remains the primary mode of transportation for older Americans (Choi et al. 2012). Driving is linked to individual well-being and driving cessation can negatively impact older adults independence and mental health (Chihuri et al. 2016; Edwards et al. 2009; Foley et al. 2002; Harmon et al. 2018). Identifying ways to support older drivers to stay on the road safely and maintain independence and community involvement is a national priority (Classen et al. 2007; NHTSA 2010; NHTSA 1999). Safety is an important consideration since older drivers are at increased risk of crashes (Classen et al. 2007; Pomidor 2016). Fatal crash rates among older drivers increase after age 75 (Cicchino 2015); generally older drivers pose a greater risk to themselves than to the community around them (Braver and Trempel 2004; Tefft 2008). Estimation of an individual driver's risk remains difficult, as on-road testing is costly and not always available (Betz et al. 2014) and office-based assessments are impractical (Betz et al. 2015) and not routine (Betz et al. 2016a).

Decisions about changing driving habits or cessation are difficult and emotionally-laden, owing to intersecting implications of safety, independence, and personal well-being. Additionally, these decisions generally involve multiple people, including the older driver and family members or trusted friends. In some instances, healthcare providers are involved at the request of family or due to concerns about safety. Older drivers fear losing their independence, being abandoned, or becoming a burden on others, while simultaneously fearing causing harm to others on the road (Betz et al. 2016b). Decisions about driving are complicated by older adults' functional ability, cognitive ability, decisional capacity, personality, and other factors such as financial resources or access to alternative transportation options. Of particular importance is understanding the impact of cognitive impairment and driving decisions. While physical function and certain medications can affect driving quality, Alzheimer's disease and other forms of progressive cognitive impairment may have the strongest link to both driving risk and the need for eventual driving cessation (Carr and Ott 2010; Pomidor 2016). Given the estimate of almost 16 million older adults in the US with dementia by 2050 (Alzheimer's Association 2020), the decision-making needs around driving for this group is significant. The role of family members, trusted friends, and healthcare providers in supporting a driver through driving retirement gains additional importance in the context of cognitive impairment or concerns about decisional capacity.

Guides and self-assessment tools exist to help older drivers and their families think about driving (AAA 2005; NIA 2014; Pomidor 2016; The Hartford 2010), but they do not include evidence-based elements that assist with values clarification and assessing the risks and benefits of driving cessation. Healthwise, a nonprofit organization that provides decision support tools and other services to enhance patient-centered decision making, released a decision aid in 2015 for US drivers with and without cognitive impairment (Healthwise 2016). The tool was developed according to international decision aid standards (Coulter et al. 2013; OHRI 2015), is available online, and is accessible to clinicians in healthcare systems who use Healthwise tools, about $25 \%$ of clinicians in the United States. However, the Healthwise decision aid has never been tested in a randomized controlled trial to evaluate its effectiveness on decision quality or person-centered outcomes.

\section{Methods}

AUTO study design

The Advancing Understanding of Transportation Options (AUTO) study is a randomized, controlled trial being conducted with older primary care patients in three US states that seeks to assess the effects of the Healthwise decision aid among older adults and a study partner (SP). AUTO will test the decision aid in improving decision making and quality and determine its effects on specific subpopulations of older drivers defined by cognitive function, 
decisional capacity, and attitudes about mobility transition. The hypothesis is that the decision aid will improve the quality of decision making about driving behaviors, which will mitigate the negative psychosocial impacts of driving reduction or cessation. The AUTO trial will enroll 300 patient-study partner dyads from primary care clinics in California, Colorado, and Indiana, USA. These dyads will be randomized into two groups $(n=150)$, stratified by site. Both members of the dyad review the same site (decision aid or control). Dyads randomized to the Healthwise driving decision aid will view the decision aid, separately, immediately following baseline assessment (Healthwise 2016). Dyads randomized to the control group view the National Institute on Aging (NIA) "Older Drivers" website (NIA 2014) immediately following the baseline assessment. Dyads in both groups are instructed to view the decision aid or control site at their own pace and navigate through the site components at their own discretion. The AUTO trial will measure the primary and secondary outcomes at baseline, 6, 12, 18 and 24 months. Consent, enrollment, data collection, and viewing the decision aid or control material is done by telephone and access to the internet; prior to the COVID-19 pandemic, it was also done face-to-face (see below).

This study protocol has followed the Standard Protocol Items: Recommendations for Interventional Trials (SPIRIT) Guidelines (Chan et al. 2013). The trial will be conducted and reported according to the reporting of pragmatic trials: an extension of the Consolidated Standards of Reporting Trials (CONSORT) Statement. The study has been approved by the institutional review boards of University of California San Diego, University of Colorado, and Indiana University. The AUTO trial is registered with clinicaltrials.gov (Clinical Trials.gov Identifier NCT04141891).
Older adult-family member dyads are recruited from primary care clinics affiliated with each study, with an enrollment goal of 100 drivers and $\geq 67$ study partners (i.e., $\geq 67$ study dyads) per site.

\section{Eligibility}

The target population is dyads formed by: (1) an adult aged 70 or older $(n=300)$ and (2) a family member, friend, or legal healthcare power of attorney whom the patient identifies as someone who might be involved in decision-making about driving or in providing support for the transition to nondriving ( $n=$ up to 300 ; Table 1 ). Eligibility for patients is established through screening of the patient's electronic health record (EHR) and by assessments conducted by the research assistants face-to-face or via the telephone. The study seeks to enroll drivers more likely to be primed to consider driving retirement, so an eligibility criterion is that that they have $\geq 1$ diagnosis of a progressive medical condition associated with reduced driving ability and increased risk of cessation, as defined by our study team (Appendix 1). These conditions were abstracted from participant's electronic medical record list of diagnoses and verbally confirmed during eligibility screening.

\section{Recruitment}

Site study coordinators obtain lists of potentially eligible older driver participants at each site and then mail recruitment letters with a site-specific recruitment flyer. If the participant has not reached out to the site, a phone call is made two weeks after the letter is sent to inquire about interested and eligible. If interested and eligible, the older driver is asked for the name and contact information of a family member and additionally requests permission to call the family member to assess if they are also eligible and willing to enroll. Eligibility screening for the

Table 1 Eligibility criteria for AUTO study

\begin{tabular}{ll}
\hline Inclusion & Exclusion \\
\hline Fluent in English & 5 -min Montreal Cognitive Assessment (MoCA) score \\
Have a telephone number for follow up interviews & $<21$ \\
Age: $\geq 70$ years (drivers); $\geq 18$ years (study partners) & In legal custody or institutionalized \\
Drivers only: & Drivers only: \\
Valid driver's license from study site's state & Currently enrolled in LongROAD longitudinal study \\
Drive at least once a week & (also at UCH and UCSD) \\
Since the last license renewal, no major changes to & \\
health, vision, or hearing that seriously impair driving & \\
(self-report) & \\
Do not feel the DMV would have serious concerns & \\
about driving (self-report) & \\
Have $\geq 1$ medical condition linked in driving cessation & \\
(EMR, confirmed by self-report; see Appendix) & \\
\hline
\end{tabular}


study partner is conducted prior to consenting the older driver. If the older driver does not identify a study partner or the study partner is ineligible or not interested, the older driver is placed on a waitlist to be contacted for enrollment once dyadic recruitment goals are met.

The AUTO trial will reduce loss to follow-up for longitudinal assessments by engaging the older drivers every 6 months throughout the study $(6,12,18$, and 24 months) and SPs every 12 months (12 and 24 months), including keeping the assignment of research staff and participants consistent at each outcome assessment and sending reminder letters.

\section{Randomization and blinding}

Enrolled older drivers are randomized in blocks to reduce bias and aim for balance among arms (Efird 2011), with randomly varied block sizes of 4 and 6 , in a 1:1 ratio of intervention to control group. The allocation is concealed using a centralized, computer-generated list that study coordinators access once the RA completes the administration of pre-randomization measures. The driver and SP are randomized to the same arm but complete study measures and interventions on their own. Participants are instructed to view the intervention or control site at their own pace and by navigating through the site components at their own discretion. After completing the decision aid or website review, all participants in both arms answer questions about knowledge, values, and driving retirement plans.

Participants are blinded to their allocation, though they know that the study is about driving. RAs cannot be blinded to the baseline assignment of SPs since SPs are given the same assignment as the driver. Whenever possible, a different RA conducts follow-up interviews to be blinded to study arm.

\section{Description of intervention}

The Healthwise decision aid is for older adults considering the decision "Is it time to stop driving?" (Healthwise 2016). The online decision aid has six sections: "Get the Facts," "Compare Options," "Your Feelings," "Your Decision," "Quiz Yourself," and "Your Summary." The decision options ("Stop driving" or "Keep driving") are presented with their benefits and risks or side effects, alongside personal stories from other adults facing the decision. The "Your Feelings" page allows users to rate on 7-point Likert scales their: concern about getting into an accident; comfort while driving; fear of harming others; concern from others; and ability and willingness to use other sources of transportation. The "Your
Decision" prompts them to rate (on a 7-point Likert scale) their current plan, from "leaning toward stopping driving" to "leaning toward keeping driving." The online DDA has a simple greyscale interface without images or videos.

\section{Description of control}

The NIA "Older Drivers" website (NIA 2014) was chosen as a control because it best represents easilyaccessible and freely-available information about driving risk and driving cessation that any older adult with internet access could view. However, the NIA website does not guide the individual through the decision-making process. It includes a personal story from an older adult, information about various medical conditions that can affect driving, and ideas of alternative transportation. It does not include videos or images.

\section{Theoretical framework}

Key measures in this trial are tied directly to the Ottawa Decision Support Framework (O'Connor 2006), which posits that decisional needs (e.g., knowledge, conflict/uncertainty, and values) affect decision quality (Fig. 1). High quality decisions, which are those both informed and reflective of the individual's values, can spur action and subsequent health outcomes and patient's feelings about the decision, such that the highest quality decision will have the best outcome for the patient. Decision aids, including the Healthwise one being evaluated in AUTO, generally have four key sections: (a) identify the decision to be made, (b) describe risks and benefits of various options, (c) assist the individual in clarifying personal values, and (d) activate the individual for decision-making (Bhandari et al. 2008; Matlock and Spatz 2014).

\section{Primary and secondary outcome measures}

The primary immediate outcome is decision conflict. Secondary, longitudinal outcomes are psychosocialand mobility-related (Fig. 1; Table 2). Primary and secondary outcome measures will be assessed at baseline, $6,12,18$, and 24 months by blinded research assistants, with planned analyses stratified by subgroups. No data is collected until informed consent are obtained. Full study measures are shown in Table 2.

\section{Primary outcome}

The primary outcome is decision quality as estimated by the Decisional Conflict Scale (DCS; Appendix 2)(O'Connor 1993; Sepucha et al. 2013). Decision quality is a fundamental element of the 


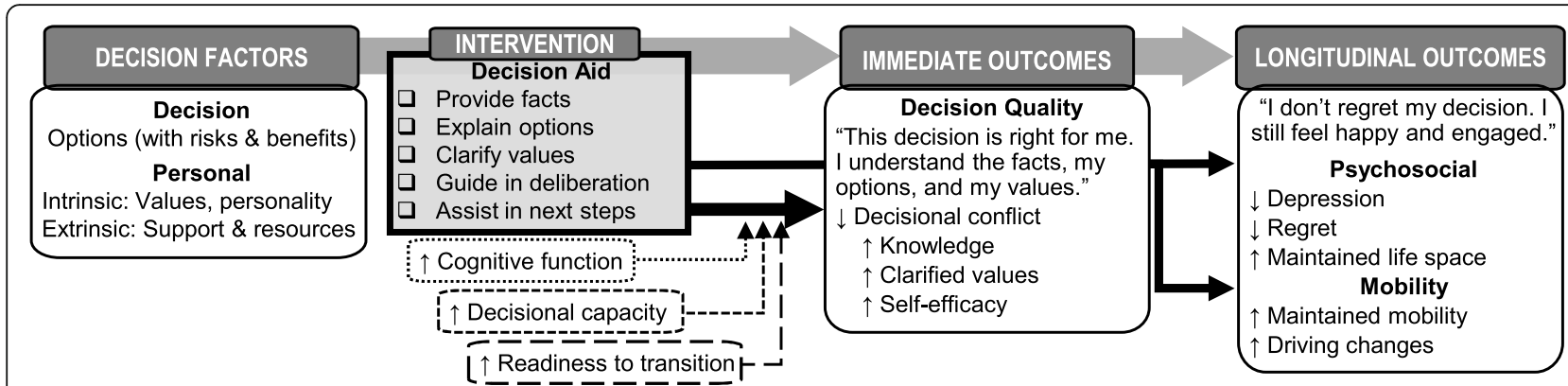

Fig. 1 Theoretical framework of decision aid's immediate and long-term impact. Adapted from Ottawa Decision Support Framework (O'Connor 2006)

Ottawa Decision Support Framework (O'Connor 2006) as a precursor to behavior change, with a high-quality decision defined as an informed patient making a decision consistent with their values (Sepucha et al. 2013). The DCS measures internal conflict or ambivalence about the decision, with higher internal conflict (or ambivalence) indicating the decision is less in-line with personal values. The DCS is a 16-item scale with three subscales, including Values Concordance. The DCS has strong reliability and test-retest correlation (Cronbach's alpha coefficients $>0.78$ ) and has previously shown to discriminate between known groups who make or delay decisions (effect size 0.4-0.8). Scores $<25$ (out of 100 total) have previously been associated with implementing decisions (O'Connor 1993). DCS scores range from 0 (extremely clear) to 100 (extremely unclear about personal values).

Secondary immediate outcomes related to decision quality are knowledge about driving decisions and decision self-efficacy. The Knowledge questionnaire, created for this study, assesses concepts about driving presented in the decision aid and control group websites. The Decision Self-Efficacy Scale will be used to measure participants' self-confidence or belief in their ability to make decisions about driving (O'Connor 1995), as decision aids can increase self-efficacy. Scores range from 0 (extremely low) to 100 (extremely high self-efficacy).

\section{Secondary outcomes}

Depressive symptoms are measured using the PROMIS 4-item scale, with higher scores indicating higher depression. All PROMIS scores are analyzed as standardized $\mathrm{T}$-scores $($ mean $=50, \mathrm{SD}=10)$ (HealthMeasures 2020).

The Ottawa Decision Regret Scale is a validated measure that correlates with decision satisfaction and conflict; it is scored from 0 to 100 , with higher scores representing higher regret (O'Connor 1996).

The Life-Space Assessment instrument (UAB Study of Aging) is a validated tool assessing recent mobility and function (Baker et al. 2003). Composite scores range from 0 (bedbound) to 120 (travel out of town every day without assistance) (Stalvey et al. 1999); scores of $\leq 60$ are correlated with lower levels of social participation and higher mortality (Phillips et al. 2015).

Driving-related measures drawn from prior studies and developed de novo include self-reported driving frequency (days per week), avoidance in certain situations (e.g., night), driving cessation (none, partial, complete), and crashes ( $\geq 1$ versus none).

\section{Cognitive measures}

Overall cognitive status is assessed through the initial screening 5-min MoCA (Wong et al. 2015), the Brief Test of Adult Cognition by Telephone (BTACT), and the Oral Trail Making Test (OTMT) (Lachman et al. 2014; Mrazik et al. 2010). A study neuropsychologist uses these cognitive tests to categorize participants' cognitive function as no impairment, mild cognitive impairment, or dementia based on the BTACT Composite and OTMT-B (zscore $<-1.5$ is impaired).

The Beck Cognitive Insight Scale combines sub-scales (self-reflectiveness and self-certainty) into a composite index, with lower scores indicating lower insight (Beck et al. 2004).

The Short Portable Assessment of Capacity for Everyday Decision-Making (SPACED) measures decision-making capacity (Lai et al. 2008). There are four criteria, each scored with a 0 for inadequate, 1 for marginal, and 2 for adequate, producing a total possible range of 0 to 8 . 
Table 2 Overall study flow for older drivers (D) and study partners (SP), following SPIRIT template of recommended content for the schedule of enrollment, interventions, and assessments (Chan et al. 2013)

\begin{tabular}{|c|c|c|c|c|c|c|c|c|}
\hline \multirow[b]{3}{*}{ TIMEPOINT (months) } & \multicolumn{8}{|c|}{ STUDY PERIOD } \\
\hline & \multirow{2}{*}{$\begin{array}{l}\text { Enrollment } \\
-t\end{array}$} & \multicolumn{2}{|c|}{ Allocation } & \multicolumn{2}{|c|}{ Post-allocation } & \multirow[b]{2}{*}{$t_{12 \mathrm{mo}}$} & \multirow[b]{2}{*}{$t_{18 \mathrm{mo}}$} & \multirow{2}{*}{$\frac{\text { Close-out }}{t_{24 \mathrm{mo}}}$} \\
\hline & & $t_{0}$ & $t_{0}$ & $t_{0}$ & $t_{6 m o}$ & & & \\
\hline \multicolumn{9}{|l|}{ ENROLLMENT } \\
\hline Eligibility screen & $X^{D, S P}$ & & & & & & & \\
\hline Informed consent & & $X^{D, S P}$ & & & & & & \\
\hline Randomization & & & $X^{D, S P}$ & & & & & \\
\hline \multicolumn{9}{|l|}{ INTERVENTIONS } \\
\hline Driving decision aid & & & $X^{D, S P}$ & & & & & \\
\hline Control website & & & $X^{D, S P}$ & & & & & \\
\hline \multicolumn{9}{|l|}{ ASSESSMENTS } \\
\hline \multicolumn{9}{|l|}{ Immediate outcomes } \\
\hline Decision Conflict Scale & & $X^{D, S P}$ & & $X^{D, S P}$ & $X^{D}$ & $X^{\mathrm{D}, S P}$ & $X^{D}$ & $X^{\mathrm{D}, \mathrm{SP}}$ \\
\hline Knowledge questionnaire & & $x^{D}$ & & $X^{\mathrm{D}, \mathrm{SP}}$ & $x^{D}$ & $X^{D, S P}$ & $X^{D}$ & $X^{\mathrm{D}, S P}$ \\
\hline Values concordance (DCS Values Clarity subscale) & & $X^{\mathrm{D}, S P}$ & & $X^{\mathrm{D}, \mathrm{SP}}$ & $x^{D}$ & $X^{\mathrm{D}, S P}$ & $x^{\mathrm{D}}$ & $X^{\mathrm{D}, \mathrm{SP}}$ \\
\hline Decision Self-Efficacy scale & & $x^{D}$ & & $X^{D, S P}$ & $x^{D}$ & $X^{D, S P}$ & $x^{D}$ & $X^{D, S P}$ \\
\hline \multicolumn{9}{|l|}{ Longitudinal outcomes } \\
\hline PROMIS Depression & & $X^{D}$ & & & $X^{D}$ & $X^{D}$ & $X^{D}$ & $X^{D}$ \\
\hline Ottawa Decision Regret Scale & & & & & $x^{D}$ & $X^{\mathrm{D}, \mathrm{SP}}$ & $X^{D}$ & $X^{\mathrm{D}, \mathrm{SP}}$ \\
\hline Life-Space Assessment & & $X^{D}$ & & & $x^{D}$ & $x^{D}$ & $X^{D}$ & $x^{D}$ \\
\hline Driving behaviors & & $x^{D}$ & & & $X^{D}$ & $X^{D, S P^{*}}$ & $X^{D}$ & $X^{D, S P^{*}}$ \\
\hline \multicolumn{9}{|l|}{ Subgroups } \\
\hline Cognitive screening (5-min MoCA) & $X^{D, S P}$ & & & & $x^{\mathrm{D}}$ & $X^{\mathrm{D}, \mathrm{SP}}$ & $X^{\mathrm{D}}$ & $X^{\mathrm{D}, \mathrm{SP}}$ \\
\hline Cognitive function (BTACT, OTMT) & & $x^{D}$ & & & & & & \\
\hline Beck Cognitive Insight Scale & & $X^{D}$ & & & & & & $X^{D}$ \\
\hline Decisional capacity (SPACED) & & $x^{\mathrm{D}}$ & & & & & & $X^{D}$ \\
\hline Attitudes about driving (ARMT) & & $X^{\mathrm{D}}$ & & & $x^{D}$ & $x^{\mathrm{D}}$ & $x^{D}$ & $X^{\mathrm{D}}$ \\
\hline Driving behaviors & & $X^{\mathrm{D}, S P}$ & & & $x^{D}$ & $X^{\mathrm{D}, S P^{*}}$ & $x^{D}$ & $X^{\mathrm{D}, \mathrm{SP} *}$ \\
\hline \multicolumn{9}{|l|}{ Other covariates } \\
\hline Demographics & & $X^{D, S P}$ & & & $X^{D}$ & $X^{\mathrm{D}, \mathrm{SP}}$ & $x^{D}$ & $X^{\mathrm{D}, \mathrm{SP}}$ \\
\hline Physical health & & $x^{D}$ & & & $X^{D}$ & $X^{D}$ & $X^{D}$ & $x^{D}$ \\
\hline Mental health & & $X^{D}$ & & & $X^{D}$ & $X^{\mathrm{D}}$ & $X^{D}$ & $X^{D}$ \\
\hline Personality (TIPI) & & $x^{D}$ & & & & & & \\
\hline Driving education & & $X^{D, S P}$ & & & $X^{D}$ & $X^{D, S P}$ & $X^{D}$ & $X^{D, S P}$ \\
\hline Family questionnaire & & $X^{S P}$ & & & & $X^{\mathrm{SP}}$ & & $X^{\mathrm{SP}}$ \\
\hline Major life events & & & & & $x^{\mathrm{D}}$ & $X^{D, S P *}$ & $x^{\mathrm{D}}$ & $X^{D, S P *}$ \\
\hline COVID-19 related health & & & & $X^{D, S P}$ & $X^{D}$ & $X^{D, S P}$ & $X^{D}$ & $X^{D, S P}$ \\
\hline
\end{tabular}

*Questions about older driver, as answered by study partner

The Attitudinal Readiness for Mobility Transitions (ARMT) measures affective and emotional aspects of present or future mobility changes associated with cessation (Meuser et al. 2013). It has four subscales: anticipatory anxiety, perceived burden, avoidance, and adverse situation (i.e., the view that mobility loss is harmful to quality of life). Each of the 24 items is rated on a 1-5 Likert scale, and higher total average scores indicate lower readiness to transition (Meuser et al. 2013). 


\section{Other measures}

Baseline and follow-up Questionnaires assess demographic characteristics and Activities of Daily Living. PROMIS measures include Global Health V1.2, Emotional Support (4-item), and Social Isolation (4item) (HealthMeasures 2020). Additional measures are the 4-item Perceived Stress Scale (Cohen et al. 1983 ) and the 10-Item Personality Inventory (Gosling et al. 2003).

Follow-up questionnaires assess exposure to print or non-study online materials about driving safety or cessation, as these may affect decisions about driving cessation, as well as major life events since last contact.

Questions drawn from the The CoRonavIruS Health Impact Survey (CRISIS V0.1) Adult Self-Report Baseline Current Form (Bromet et al. 2021) were added to the questionnaires in spring 2020 to assess how the COVID19 pandemic was affecting participants' well-being and mobility. Study staff also began tracking relevant local orders that might limit driving (e.g., stay-at-home orders).

At baseline and follow-up, SPs complete additional questions about their relationship with the driver.

\section{Data monitoring}

The data safety monitoring plan (DSMP) for this trial includes monitoring by the PI and a Data Safety and Monitoring Board (DSMB). The DSMB Charter contains a detailed list of the DSMB responsibilities. The DSMB will act in an advisory capacity to the IRB and NIA Program Official in order to monitor participant safety, evaluate the progress of the study, and review procedures for data management and analysis, maintaining the confidentiality of data, and the quality of data collection.

Potential adverse events that will be monitored in the AUTO study include: death (any reason), inpatient hospitalization (any reason), emergency department visit (any reason), or motor vehicle crash. Each adverse event is graded by severity and relationship to intervention.

Given concerns over the safety of older drivers and the community around them, the AUTO team developed specific procedures related to potentiallyimpaired drivers. For older drivers with an initial 5min MoCA score of 21-25 (Additional file 1), study staff review questionnaire elements related to the American Academy of Neurology guidelines (Iverson et al. 2010). If the driver has $\geq 3$ risk factors (e.g., reported crashes), the site PI contacts the driver to recommend they have a professional driving evaluation and talk with their primary care provider. The driver is allowed to continue participation in the study whether or not they complete a driving evaluation. Additionally, AUTO staff can note concerns from participant interactions, such as confusion with questions or suspected memory problems in participants. These concerns similarly prompt an ad hoc review and potential contact of the older driver by the site PI with a recommendation to talk to their physician or trusted family or friends (Additional file 2). All these procedures are explained during the informed consent process. At the time of consent, older drivers are asked if they want to provide optional consent for the research team to contact their primary care provider or a designated friend or family member about their driving safety, should the team have concerns about safety.

\section{Data collection}

Before March 2020, RAs met with older drivers to complete the baseline visits in person at on-campus sites or participants' homes. The baseline session includes: written informed consent including HIPAA authorization and permission to access driver licensing records (crash records for the past 12 months before enrollment and up to every 12 months after enrollment); administered questionnaires before and after randomization; brief cognitive tests; and viewing intervention or control information on a tablet. Study measures and point of administration are shown in Fig. 1. Procedures for SPs are similar, although SPs could complete their baseline session either in-person or over the phone. Response cards were used to help facilitate the various questionnaires.

The COVID-19 pandemic and campus closures prompted a change in the study protocol such that all older driver and SP enrollments are done by phone. Baseline sessions completed by telephone (unless the participant requested Zoom videoconference) use a postcard consent with a waiver of written documentation. Response cards and a link to the website (decision aid or control) are emailed to participants, with instructions to view them on the participant's desktop, laptop, tablet, or smart phone.

Participants are contacted for telephone follow-up at pre-specified intervals: $6,12,18$, and 24 months after initial visit for drivers; 12 and 24 months after initial visit for SPs. Participants are contacted via phone call or email (depending on participants' preference) to schedule the follow-up interviews. Trained research staff follow all institutional review board (IRB) policies regarding contacting participants, with at least 3 but no more than 10 contact attempts for non-responders at each follow-up interval. Research staff attempt to call participants at different times of day, leaving brief messages on 
varying attempts, and contact participants via email and mail as needed.

RAs at each site enter all data into a secure research database (REDCap, Research Electronic Data Capture) (Harris et al. 2009). Data access privileges specify only those privileges required by each individual in their specific organizational role, so RAs can only see their site's data, and limited study team members (PI, biostatistician, and analyst) can see data from all sites. All study documents are stored in locked cabinets or password-protected files on secure university servers. Recruitment, enrollment, and follow-up reports are sent weekly to research staff, coordinators, and co-investigators to allow monitoring of study progress.

\section{Timeline}

The recruitment of patients began in December 2019, with anticipated enrollment completion by June 2021 and all data collected from recurring outcome assessments expected to be collected by June 2023.

\section{Analysis plan}

Planned analyses will be performed according to the principle of intention-to-treat, including all randomized drivers. Unless otherwise specified, hypothesis tests will be two-sided with alpha $=.05$, with $95 \%$ confidence intervals or $p$ values reported. Descriptive statistics will be computed for baseline patient characteristics, initially testing for differences between control and intervention groups. Site effects will be assessed by comparing demographic variables across the sites, using one-way ANOVA tests for continuous measures and chi-square tests of proportions for categorical measures. If there are significant differences among sites, then (assuming enough events) analysis will use separate models for each aim described below, with a fixed effect for site and with treatment arm as the main predictor. For longitudinal analyses, continuous and logistic outcomes will be modeled using generalized estimating equations with unstructured correlation structure to account for repeated observations on each participant. Analyses of the decision aid's longitudinal effects may be vulnerable to bias, as control arm participants may be exposed to intervention arm messages through exposure to available materials or courses related to driving safety or cessation (e.g., websites, physician counseling). Analyses may adjust measurements of contamination as applicable. In all analyses, validation of distributional and parameterization assumptions will be checked and data transformations (e.g. log-transformations) or alternative methods will be implemented as appropriate.

Planned analyses will first test the effect of a webbased decision aid as compared to control (webbased information only) on: (a) immediate decision quality, hypothesizing that more decision aid participants will make high-quality decisions; (b) longitudinal psychosocial outcomes at 12 and 24 months, hypothesizing that decision aid participants will have reduced prevalence of depressive symptoms and of decision regret but maintained life space; and (c) longitudinal driving behaviors at 12 and 24 months, hypothesizing that the decision aid-although not intended to direct participants to continue or stop driving-will lead to changes. Next, stratified analyses will determine the decision aid's effects in specific subpopulations, including: (a) older drivers with versus without cognitive impairment, hypothesizing that the decision aid will improve decision quality more in cognitively intact drivers; (b) older drivers with maintained versus impaired decisional capacity, hypothesizing that the decision aid will improve decision quality more in drivers with maintained decisional capacity; and (c) older drivers who are attitudinally more versus less ready for a mobility transition, hypothesizing that the decision aid will improve decision quality more in drivers who are ready for transition.

Study partner data will be analyzed for the primary outcome (DCS) with regression models that allow assessment of marginal effects of the intervention on older drivers and partners separately while jointly accounting for the correlation between drivers and partners, as well as their concordance/ discordance in decision quality. We will also examine the degree of concordance (or discordance) within driver-partner dyads on various measures, including current versus desired level of partner involvement in driving retirement process (part of the driving questionnaire).

Study scales and variables were chosen carefully to keep questionnaires as short as possible and minimize overlap. To avoid inflation of our type I error rate, we selected DCS as our single primary endpoint that will be tested to assess the overall efficacy of the intervention. Other secondary outcomes (as listed above) will be considered subsidiary and exploratory rather than confirmatory. ( $\mathrm{Li}$ et al. 2017b).

\section{Sample size and power analysis}

The target sample size $(n=300 ; 150$ intervention and 150 control) was chosen to allow detection of a $20-40 \%$ difference between the decision aid and 
control arms (depending on underlying proportions for each of the treatment arms) for the primary outcome (DCS score $<25$ ) at a power of $90 \%$ and a 0.05 significance level, while allowing for $10 \%$ loss to follow-up. The overall sample size also allows for stratified analyses. Estimates for the effect of the driving decision aid on behavior among older drivers do not exist, so the assumption of a $20-40 \%$ difference is conservative (prior work with other DAs has found an effect size of $40-80 \%$ on DCS between groups) (O'Connor 1993).

\section{Discussion}

The AUTO study is the first large-scale trial in the United States for a decision aid for driving retirement among older adults. Its randomized design allows examination of the effect of a decision aid on both immediate and longterm outcomes, its multi-site recruitment will improve generalizability, and its longitudinal follow-up will complement existing observational studies of driving behaviors among older adults, like the LongROAD study and Candrive/Ozcandrive (Li et al. 2017a; Marshall et al. 2013).

The COVID-19 pandemic arose shortly after study enrollment began, prompting a shift to completely remote enrollment as of March 2020. This posed certain logistic challenges related to the length of the interview (which was more tiring for participants by telephone than in person) and technical difficulties in setting up the response cards and intervention on a home device during the baseline phone call. An additional challenge was that during the period of remote enrollment, a greater proportion of potential participants did not answer phone calls, perhaps because the call-back number is not identified as a university-affiliated number. However, the shift to remote study activities brought positive effects as well. This included a more rapid rate of enrollment and high rates of participation among those eligible, perhaps related to the desire of older adults to be engaged during times of social distancing or quarantine (Fuller and Huseth-Zosel, 2021).

The nature of the study raised a unique challenge related to the tension between study integrity and participant safety. The study seeks to examine the factors affecting an older adult's decision about driving, with a desire to avoid providing information, guidance, or recommendations to participants (other than the decision aid itself for the group). Yet responses to study measures or interactions with staff might uncover cognitive or physical issues that have the potential to impair driving ability, thereby posing a risk to the participant and those around them. In addition, the study specifically seeks to enroll older adults with at least one medical condition (including mild cognitive impairment) that might contribute to driving retirement so as to allow an adequate sample of older adults who stop driving during the study. The AUTO team, in consultation with its DSMB, developed study protocols and safety checks to protect participant safety and well-being without overly interfering in decision-making about driving retirement.

Additional study limitations include challenges, and potential biases, in enrolling study dyads. Specifically, some older adults may not have or want to suggest a family member or friend to participate as a study partner, and the study design may not allow a large enough sample of these older drivers (without partners) for detailed subgroup analyses. We chose to allow some older adults to enroll without a partner out of recognition that their experiences and outcomes may differ from those with a partner, and the study will provide at least preliminary analyses. We chose to have participants and partners complete the decision aid separately, so we could measure their responses separately (and potential concordance); in real life, such tools may be used together. The COVID-19 pandemic itself affected older drivers' behaviors (Morrow-Howell et al. 2020; Rantanen et al. 2020), with reduced frequency and distances driven and anxieties about the safety of public transportation, which might impact the study's analyses and outcomes. Added study questions about COVID-19 and its effects on mobility and on mental and physical health will allow examination of some of these issues. In addition, internet access was needed to view the informational website and response option cards in real time, which may have affected participant diversity.

\section{Conclusion}

The AUTO study has the potential to help fill knowledge gaps concerning decision making about driving retirement and to advance the science and practice of safe mobility through a life-course perspective. The innovative application of the decision aid model to older driver decision making offers the possibility of facilitating decisions about driving retirement in a person-centered, acceptable, feasible way, and consequently it has the potential to reduce the negative psychosocial outcomes associated with driving retirement. Understanding whether and with whom to use a driving decision aid has the potential to significantly improve the independence, health, and well-being of millions of older adults. 


\section{Appendix 1}

Table 3 Medical conditions, as identified in electronic medical record, with $\geq 1$ required for older driver eligibility

\section{Medical Condition}

Diseases/conditions affecting vision

Cardiovascular disease, especially when associated with presyncope, syncope, or cognitive deficits

Neurologic disease

Psychiatric disease

Metabolic disease

Musculoskeletal disabilities

Respiratory disease

Chronic renal failure

Insomnia
Examples

Diabetic retinopathy

Macular degeneration

Glaucoma

Retinitis pigmentosa

Field cuts

Low visual acuity even after correction

Unstable coronary syndrome

Implantable defibrillator

Congestive heart failure

Hypertrophic obstructive cardiomyopathy

Orthostatic hypotension

Syncope or presyncope

Narcolepsy

Dementia

Multiple sclerosis

Parkinson disease

Brain injury

Spinal cord injury

Stroke

Vertigo or dizziness

Seizure

Alcohol or other substance abuse

IDDM

Arthritis and foot abnormalities

Chronic obstructive pulmonary disease

Obstructive sleep apnea

End Stage Renal Disease

Hemodialysis

Sleep apnea

Insomnia

Restless leg syndrome 


\section{Appendix 2}

\section{Decision Conflict Scale (DCS) (O'Connor 1993)}

When you think about driving, which way are you leaning? Please use a 1 to 7 scale where 1 is Leaning Toward Stop Driving, 4 is Undecided, and 7 is Leaning Toward Continue Driving.

\begin{tabular}{lcccccc}
\hline Stop Driving & & & & & $\begin{array}{c}\text { Continue } \\
\text { Driving }\end{array}$ \\
$\mathbf{1}$ & $\mathbf{2}$ & $\mathbf{3}$ & $\mathbf{4}$ & $\mathbf{5}$ & $\mathbf{6}$ & $\mathbf{7}$ \\
\hline Leaning & & & Undecided & & & Leaning \\
Toward & & & & & & Toward \\
\hline
\end{tabular}

In thinking about your preference for whether to stop or continue driving, please indicate how strongly you disagree or agree with the following statements using a 1 to 5 scale where 1 is Strongly Disagree and 5 is Strongly Agree.

\begin{tabular}{|c|c|c|c|c|c|c|}
\hline Statement & $\begin{array}{l}\text { Strongly } \\
\text { Disagree }\end{array}$ & Disagree & $\begin{array}{l}\text { Neither } \\
\text { Agree } \\
\text { Nor } \\
\text { Disagree }\end{array}$ & Agree & $\begin{array}{l}\text { Strongly } \\
\text { Agree }\end{array}$ & $\begin{array}{l}\text { Don't } \\
\text { Know }\end{array}$ \\
\hline $\begin{array}{l}\text { I know which } \\
\text { options are } \\
\text { available when it } \\
\text { comes to } \\
\text { stopping or } \\
\text { continuing } \\
\text { driving. }\end{array}$ & 1 & 2 & 3 & 4 & 5 & 88 \\
\hline $\begin{array}{l}\text { I know the } \\
\text { benefits of } \\
\text { stopping or } \\
\text { continuing } \\
\text { driving. }\end{array}$ & 1 & 2 & 3 & 4 & 5 & 88 \\
\hline $\begin{array}{l}\text { I know the risks } \\
\text { or drawbacks of } \\
\text { stopping or } \\
\text { continuing } \\
\text { driving. }\end{array}$ & 1 & 2 & 3 & 4 & 5 & 88 \\
\hline $\begin{array}{l}\text { I am clear about } \\
\text { which benefits } \\
\text { matter most to } \\
\text { me. }\end{array}$ & 1 & 2 & 3 & 4 & 5 & 88 \\
\hline $\begin{array}{l}\text { I am clear about } \\
\text { which risks or } \\
\text { drawbacks } \\
\text { matter most to } \\
\text { me. }\end{array}$ & 1 & 2 & 3 & 4 & 5 & 88 \\
\hline $\begin{array}{l}\text { I am clear about } \\
\text { which is more } \\
\text { important to me } \\
\text { (the benefits or } \\
\text { the risks and } \\
\text { drawbacks). }\end{array}$ & 1 & 2 & 3 & 4 & 5 & 88 \\
\hline $\begin{array}{l}\text { I have enough } \\
\text { support from } \\
\text { others to make a } \\
\text { choice when it } \\
\text { comes to } \\
\text { stopping or } \\
\text { continuing. }\end{array}$ & 1 & 2 & 3 & 4 & 5 & 88 \\
\hline $\begin{array}{l}\text { I am choosing to } \\
\text { either stop or } \\
\text { continue driving } \\
\text { without pressure } \\
\text { from others. }\end{array}$ & 1 & 2 & 3 & 4 & 5 & 88 \\
\hline
\end{tabular}

Decision Conflict Scale (DCS) (O'Connor 1993) (Continued)

\begin{tabular}{|c|c|c|c|c|c|c|}
\hline Statement & $\begin{array}{l}\text { Strongly } \\
\text { Disagree }\end{array}$ & Disagree & $\begin{array}{l}\text { Neither } \\
\text { Agree } \\
\text { Nor } \\
\text { Disagree }\end{array}$ & Agree & $\begin{array}{l}\text { Strongly } \\
\text { Agree }\end{array}$ & $\begin{array}{l}\text { Don't } \\
\text { Know }\end{array}$ \\
\hline $\begin{array}{l}\text { I have enough } \\
\text { advice to make a } \\
\text { choice to stop or } \\
\text { continue driving. }\end{array}$ & 1 & 2 & 3 & 4 & 5 & 88 \\
\hline $\begin{array}{l}\text { I am clear about } \\
\text { the best choice } \\
\text { for me. }\end{array}$ & 1 & 2 & 3 & 4 & 5 & 88 \\
\hline $\begin{array}{l}\text { I feel sure about } \\
\text { what to choose } \\
\text { from. }\end{array}$ & 1 & 2 & 3 & 4 & 5 & 88 \\
\hline $\begin{array}{l}\text { The decision to } \\
\text { stop or continue } \\
\text { driving is easy } \\
\text { for me to make. }\end{array}$ & 1 & 2 & 3 & 4 & 5 & 88 \\
\hline $\begin{array}{l}\text { I feel I have } \\
\text { made an } \\
\text { informed choice. }\end{array}$ & 1 & 2 & 3 & 4 & 5 & 88 \\
\hline $\begin{array}{l}\text { My decision } \\
\text { shows what is } \\
\text { important to me. }\end{array}$ & 1 & 2 & 3 & 4 & 5 & 88 \\
\hline $\begin{array}{l}\text { I expect to stick } \\
\text { with my decision } \\
\text { to stop or } \\
\text { continue driving. }\end{array}$ & 1 & 2 & 3 & 4 & 5 & 88 \\
\hline $\begin{array}{l}\text { I am satisfied } \\
\text { with my decision } \\
\text { to stop or } \\
\text { continue driving. }\end{array}$ & 1 & 2 & 3 & 4 & 5 & 88 \\
\hline
\end{tabular}

\section{Supplementary Information}

The online version contains supplementary material available at https://doi. org/10.1186/s40621-021-00310-4.

Additional file 1: Risk Review Form (based on AAN Guidelines

(Iverson et al. 2010).

Additional file 2: Cognitive Concern Form

\begin{abstract}
Abbreviations
AUTO: Advancing Understanding of Transportation Options; DCS: Decisional Conflict Scale; DSMB: Data Safety and Monitoring Board; IRB: Institutional Review Board; MoCA: Montreal Cognitive Assessment; NIA: National Institute on Aging; RA: Research Assistant; SP: Study Partner
\end{abstract}

\section{Acknowledgements}

Additional members of the AUTO Research Team: Miles Breese, Abigail Evans, Alexa Hansen, Eleanor Batista-Malat, Natalie Moursund, Alicia Okimura, Sarah Andrade, Eugenia Orehova, Shelley Suarez, Ruby Vianzon, and Anna Williams.

Authors' contributions

Study concept and design: MEB, CGD, NF, DSH, LH, CEK, and DDM. Study instruments and research protocols: MEB, FO, LM, CGD, NF, DSH, LH, RJ, CEK, and DDM. Pilot testing: MEB, FO, LM, NF, DSH, RJ, and LH. Recruitment and Enrollment: MEB, FO, LM, NF, LH, and RM. Data systems: LM and RJ. Quality assurance: MEB, LM, DSH, and RJ. Study oversight: MEB, LM, NF, and LH. Manuscript: MEB and FO drafted the manuscript, and all authors contributed to the critical revision of the manuscript and approved the final manuscript. The authors read and approved the final manuscript. 


\section{Funding}

This project was supported by NIH/NIA Grant Number R01 AG059613 and by NIH/NCATS Colorado CTSA Grant Number UL1 TR002535. The contents of this work are the authors' sole responsibility and do not necessarily represent official funder views or the views of the Department of Veterans Affairs.

\section{Availability of data and materials}

Requests for data from other researchers and the public will be considered, and data will be made available in accordance with local institution policies, IRB recommendations, local/state/federal laws and regulations, and considerations for publication. Any applicable data sharing will follow HIPAA rules.

\section{Ethics approval and consent to participate}

All study procedures and documents were reviewed and approved under Expedited Review, Category 7, by the Colorado Multiple Institutional Review Board (COMIRB). COMIRB served as the central IRB for the study. COMIRB approved a waiver of written documentation of informed consent and HIPAA authorization; the protocol included a comprehensive oral consent process.

\section{Consent for publication}

\author{
Not applicable.
}

\section{Competing interests}

The authors declare that they have no competing interests.

\section{Author details}

${ }^{1}$ Department of Emergency Medicine, School of Medicine, University of Colorado Anschutz Medical Campus, Aurora, CO, USA. ${ }^{2}$ VA Eastern Colorado Geriatric Research Education and Clinical Center, Aurora, CO, USA.

${ }^{3}$ Department of Epidemiology, Colorado School of Public Health, University of Colorado Anschutz Medical Campus, Aurora, CO, USA. ${ }^{4}$ Center for Aging Research, Indiana University School of Medicine, Regenstrief Institute, Indianapolis, IN, USA. ${ }^{5}$ Department of Family Medicine, University of Southern California, Los Angeles, CA, USA. 'School of Public Health, University of California San Diego, San Diego, CA, USA. ${ }^{7}$ Department of Biostatistics and Informatics, Colorado School of Public Health, University of Colorado Anschutz Medical Campus, Aurora, CO, USA. ${ }^{8}$ Adult \& Child Consortium for Outcomes Research \& Delivery Science, School of Medicine, University of Colorado Anschutz Medical Campus, Aurora, CO, USA. ${ }^{9}$ Division of Cardiology, School of Medicine, University of Colorado Anschutz Medical Campus, Aurora, CO, USA. ${ }^{10}$ Division of Geriatric Medicine, Department of Medicine, University of Colorado School of Medicine, Aurora, CO, USA.

\section{Received: 10 December 2020 Accepted: 16 February 2021}

\section{Published online: 03 May 2021}

\section{References}

AAA. AAA Roadwise Review: A tool to help seniors drive safely longer. American Automobile Association. 2005. http://www.aaaexchange.com/MAIN/Default.a sp? CategorylD=3\&SubCategorylD=38\&ContentID=315. Accessed 5 Feb 2021.

Alzheimer's Association. 2020 Alzheimer's disease facts and figures 2020. https:// www.alz.org/media/Documents/alzheimers-facts-and-figures.pdf. Accessed 5 Feb 2021.

Baker PS, Bodner EV, Allman RM. Measuring life-space mobility in communitydwelling older adults. J Am Geriatr Soc. 2003;51(11):1610-4.

Beck AT, Baruch E, Balter JM, Steer RA, Warman DM. A new instrument for measuring insight: the Beck cognitive insight scale. Schizophr Res. 2004;68(23):319-29.

Betz ME, Dickerson A, Coolman T, Schold Davis E, Jones J, Schwartz R. Driving rehabilitation programs for older drivers in the United States. Occup Ther Health Care. 2014;28(3):306-17.

Betz ME, Jones J, Carr DB. System facilitators and barriers to discussing older driver safety in primary care settings. Inj Prev. 2015;21(4):231-7.

Betz ME, Kanani H, Juarez-Colunga E, Schwartz R. Discussions about driving between older adults and primary care providers. J Am Geriatr Soc. 2016a; 64(6):1318-23.

Betz ME, Scott K, Jones J, Diguiseppi C. "Are you still driving?" Metasynthesis of patient preferences for communication with health care providers. Traffic Inj Prev. 2016b;17(4):367-73.
Bhandari G, Hassanein K, Deaves R. Debiasing investors with decision support systems: an experimental investigation. Decis Support Syst. 2008;46(1):399_ 410

Braver ER, Trempel RE. Are older drivers actually at higher risk of involvement in collisions resulting in deaths or non-fatal injuries among their passengers and other road users? Inj Prev. 2004;10(1):27-32.

Bromet E, Colcombe S, Geogiadis K, Klein D, Salum G. The CoRonavlruS Health Impact Survey (CRISIS): adult self-report baseline current form. https://www. nIm.nih.gov/dr2/CRISIS_Adult_Self-Report_Baseline_Current_Form_V0.3.pdf. Accessed 5 Feb 2021

Carr DB, Ott BR. The older adult driver with cognitive impairment: "It's a very frustrating life". JAMA. 2010;303(16):1632-41.

CDC. Older adult drivers. 2020. https://www.cdc.gov/transportationsafety/older_a dult_drivers/index.html. Accessed 5 Feb 2021.

Chan A-W, Tetzlaff JM, Altman DG, Laupacis A, Gøtzsche PC, Krleža-Jerić K, et al. SPIRIT 2013 statement: defining standard protocol items for clinical trials. Ann Intern Med. 2013;158(3):200-7.

Chihuri S, Mielenz TJ, DiMaggio CJ, Betz ME, DiGuiseppi C, Jones VC, et al. Driving cessation and health outcomes in older adults. J Am Geriatr Soc. 2016;64(2): 332-41.

Choi M, Adams KB, Kahana E. The impact of transportation support on driving cessation among community-dwelling older adults. J Gerontol B Psychol Sci Soc Sci. 2012;67B(3):392-400.

Cicchino JB. Why have fatality rates among older drivers declined? The relative contributions of changes in survivability and crash involvement. Accid Anal Prev. 2015;83:67-73.

Classen S, Lopez ED, Winter S, Awadzi KD, Ferree N, Garvan CW. Populationbased health promotion perspective for older driver safety: conceptual framework to intervention plan. Clin Interv Aging. 2007;2(4):677-93.

Cohen S, Kamarck T, Mermelstein R. A global measure of perceived stress. J Health Soc Behav. 1983;24(4):385-96.

Coulter A, Stilwell D, Kryworuchko J, Mullen PD, Ng CJ, van der Weijden T. A systematic development process for patient decision aids. BMC Med Inf Decis Mak. 2013;13(Suppl 2):S2.

Edwards JD, Perkins M, Ross LA, Reynolds SL. Driving status and three-year mortality among community-dwelling older adults. J Gerontol Biol Sci Med Sci. 2009;64(2):300-5.

Efird J. Blocked randomization with randomly selected block sizes. Int J Env Res Public Health. 2011;8(1):15-20.

Foley DJ, Heimovitz HK, Guralnik JM, Brock DB. Driving life expectancy of persons aged 70 years and older in the United States. Am J Public Health. 2002;92(8): 1284-9.

Fuller HR, Huseth-Zosel A. Lessons in resilience: initial coping among older adults during the COVID-19 pandemic. Gerontologist. 2021;61(1):114-25.

Gosling SD, Rentfrow PJ, Swann WB. A very brief measure of the big-five personality domains. J Res Personal. 2003;37(6):504-28.

Harmon A, Babulal G, Vivoda J, Zikmund-Fisher B, Carr D. Planning for a nondriving future: behaviors and beliefs among middle-aged and older drivers. Geriatrics. 2018;3(2):19.

Harris PA, Taylor R, Thielke R, Payne J, Gonzalez N, Conde JG. Research electronic data capture (REDCap)--a metadata-driven methodology and workflow process for providing translational research informatics support. J Biomed Inform. 2009;42(2):377-81.

HealthMeasures. PROMIS adult measures. 2020. http://www.healthmeasures.net/ explore-measurement-systems/promis/intro-to-promis/list-of-adult-measures. Accessed 5 Feb 2021

Healthwise. Healthy aging: is it time to stop driving?. Healthwise. 2016. https:// www.healthwise.net/cochranedecisionaid/Content/StdDocument.aspx? DOCHWID=zx3961. Accessed 5 Feb 2021.

Iverson DJ, Gronseth GS, Reger MA, Classen S. Practice parameter update: evaluation and management of driving risk in dementia. Neurology. 2010; 74(16):1316-24.

Lachman ME, Agrigoroaei S, Tun PA, Weaver SL. Monitoring cognitive functioning: psychometric properties of the brief test of adult cognition by telephone (BTACT). Assessment. 2014;21(4):404-17.

Lai JM, Gill TM, Cooney LM, Bradley EH, Hawkins KA, Karlawish JH. Everyday decision-making ability in older persons with cognitive impairment. Am J Geriatr Psychiatry. 2008;16(8):693-6.

Li G, Eby DW, Santos R, Mielenz TJ, Molnar LJ, Strogatz D, et al. Longitudinal research on aging drivers (LongROAD): study design and methods. Inj Epidemiol. 2017a;4(1):22. 
Li G, Taljaard M, Van den Heuvel ER, Levine MA, Cook DJ, Wells GA, et al. An introduction to multiplicity issues in clinical trials: the what, why, when and how. Int J Epidemiol. 2017b;46(2):746-55.

Marshall SC, Man-Son-Hing M, Bedard M, Charlton J, Gagnon S, Gelinas I, et al. Protocol for Candrive II/Ozcandrive, a multicentre prospective older driver cohort study. Accid Anal Prev. 2013;61:245-52.

Matlock DD, Spatz ES. Design and testing of tools for shared decision making Circ Cardiovasc Qual Outcomes. 2014;7(3):487-92.

Meuser TM, Berg-Weger M, Chibnall JT, Harmon AC, Stowe JD. Assessment of readiness for mobility transition (ARMT): a tool for mobility transition counseling with older adults. J Appl Gerontol. 2013;32(4):484-507.

Morrow-Howell N, Galucia N, Swinford E. Recovering from the COVID-19 pandemic: a focus on older adults. J Aging Soc Policy. 2020;32(4-5):526-35.

Mrazik M, Millis S, Drane DL. The oral trail making test: effects of age and concurrent validity. Arch Clin Neuropsychol. 2010;25(3):236-43.

NHTSA. Safe mobility for older people. National Highway Traffic Safety Administration, US Department of Transportation, DOT HS 808 853. 1999. http://www.nhtsa.dot.gov/people/injury/olddrive/safe. Accessed 5 Feb 2021.

NHTSA. Older driver program five-year strategic plan: 2012-2017. Washsington: National Highway Traffic Safety Administration, DOT HS 811 432. 2010. http:// www.nhtsa.gov/staticfiles/nti/pdf/811432.pdf. Accessed 5 Feb 2021.

NIA. Age page: older drivers. National Institute on Aging. 2014. http://www.nia. nih.gov/health/publication/older-drivers. Accessed 5 Feb 2021.

O'Connor AM. User manual - decisional conflict scale [updated 2010]. Ottawa: Ottawa Hospital Research Institute. 1993. https://decisionaid.ohri.ca/docs/ develop/User_Manuals/UM_Decisional_Conflict.pdf. Accessed 5 Feb 2021.

O'Connor AM. Decision self-efficacy scale. 1995. https://decisionaid.ohri.ca/docs/ develop/Tools/Decision_SelfEfficacy.pdf. Accessed 5 Feb 2021.

O'Connor AM. Decision regret scale. Ottawa Hospital Research Institute 1996. https://decisionaid.ohri.ca/docs/develop/User_manuals/UM_Regret_Scale.pdf. Accessed 5 Feb 2021.

O'Connor AM. Ottawa decision support framework to address decisional conflict. 2006. https://decisionaid.ohri.ca/docs/develop/ODSF.pdf. Accessed 5 Feb 2021.

OHRI. Decision aid summary (healthy aging: is it time to stop driving?). Ottawa Hospital Research Institute. 2015. https://decisionaid.ohri.ca/AZsumm.php? ID=1342. Accessed 5 Feb 2021.

Phillips J, Dal Grande E, Ritchie C, Abernethy AP, Currow DC. A population-based cross-sectional study that defined normative population data for the lifespace mobility assessment-composite score. J Pain Symptom Manag. 2015; 49(5):885-93.

Pomidor A. Clinician's guide to assessing and counseling older drivers (3rd edition). American Geriatrics Society and National Highway Traffic Safety Administration 2016. https://www.americangeriatrics.org/programs/olderdriver-safety. Accessed 5 Feb 2021.

Rantanen T, Eronen J, Kauppinen M, Kokko K, Sanaslahti S, Kajan N, et al. Lifespace mobility and active aging as factors underlying quality of life among older people before and during COVID-19 lock-down in Finland - a longitudinal study. J Gerontol A Biol Sci Med Sci. 2020:glaa274.

Sepucha KR, Borkhoff CM, Lally J, Levin CA, Matlock DD, Ng CJ, et al. Establishing the effectiveness of patient decision aids: key constructs and measurement instruments. BMC Med Inf Decis Mak. 2013;13(Suppl 2):S12.

Stalvey BT, Owsley C, Sloane ME, Ball K. The life space questionnaire: a measure of the extent of mobility of older adults. J Appl Gerontol. 1999;18(4):460-78.

Tefft BC. Risks older drivers pose to themselves and to other road users. J Saf Res. 2008;39(6):577-82.

The Hartford. We need to talk: family conversations with older drivers. The Hartford 2010. http://www.thehartford.com/mature-market-excellence/familyconversations-with-older-drivers. Accessed 5 Feb 2021.

Wong A, Nyenhuis D, Black SE, Law LS, Lo ES, Kwan PW, et al. Montreal cognitive assessment 5-minute protocol is a brief, valid, reliable, and feasible cognitive screen for telephone administration. Stroke. 2015;46(4):1059-64.

\section{Publisher's Note}

Springer Nature remains neutral with regard to jurisdictional claims in published maps and institutional affiliations.

Ready to submit your research? Choose BMC and benefit from:

- fast, convenient online submission

- thorough peer review by experienced researchers in your field

- rapid publication on acceptance

- support for research data, including large and complex data types

- gold Open Access which fosters wider collaboration and increased citations

- maximum visibility for your research: over $100 \mathrm{M}$ website views per year

At BMC, research is always in progress.

Learn more biomedcentral.com/submissions 